\title{
Spin-glass model of QCD near saturation
}

\author{
Robi Peschansk* \\ Service de physique théorique, CEA/Saclay, 91191 Gif-sur-Yvette cedex, France ${ }^{\dagger}$
}

\begin{abstract}
We establish a connection between the cascading of gluon momenta modeled within the diffusive approximation of the Balitsky-Fadin-Kuraev-Lipatov kernel and the thermodynamics of directed polymers on a tree with disorder. Using known results on the low-temperature spin-glass phase of this statistical mechanic problem we describe the dynamical phase space of gluon transverse momenta near saturation including its fluctuation pattern. It exhibits a nontrivial clustering structure, analoguous to "hot spots", whose distributions are derived and possess universal features in common with other spin-glass systems.
\end{abstract}

1. Saturation in QCD is expected to occur when parton densities inside an hadronic target are so high that their wave-functions overlap. This is expected from the high-energy evolution of deep-inelastic scattering governed by the Balitsky Fadin Kuraev Lipatov (BFKL) kernel [1]. The BFKL evolution equation is such that the number of gluons of fixed size increases exponentially and would lead, if not modified, to an average violation of unitarity. Saturation [2, 3] is characterized by a typical transverse momentum scale $Q_{s}(Y)$, depending on the overal rapidity of the reaction, at which the unitarity bound is reached by the BFKL evolution of the amplitude. The problem we want to address here is the characterization of the gluon-momentum distribution near saturation. This study may help analyzing the Color Glass Condensate (CGC) which has been proposed [4] as a description of the QCD phase at full saturation. As we shall see, our aim in the present paper is to fully understand the spectrum of transverse momentum fluctuations among the gluons which are generated by the BFKL evolution in rapidity, near saturation.

Indeed, one of the major recent challenges in QCD saturation is the problem of taking into account the rôle of fluctuations which are present in the BFKL evolution, i.e. in the dilute regime. Quite surprisingly, the fluctuations in this region modify the overall approach to saturation by a mechanism which has been understood as the formation of traveling-wave solutions of nonlinear differential equations. If at first one neglects the fluctuations (in the mean-field approximation), the effect of saturation on a dipole-target amplitude is described by the nonlinear Balitsky-Kovchegov 5] (BK) equation, where a nonlinear damping term adds to the BFKL equation. As shown in 6], this equation falls into the universality class of the Fisher and Kolmogorov Petrovsky Piscounov (F-KPP) nonlinear equation [7] which admits asymptotic traveling-wave solutions of "pulled-front" type [8]. In the QCD case, this means that they are driven by the gluons of higher momentum. Hence the overall behaviour of the solution is driven by the high-momentum tail, which is precisely in the "dilute" domain of the BFKL regime, far from the range which is dominated by saturation. The exponential behaviour of the BFKL evolution quickly enhances the effects of the tail towards a region where finally the nonlinear damping regulates both the traveling-wave propagation and structure.

In these conditions it was realized, for "pulled-front" traveling waves [9] and in QCD [10], that the fluctuations inherent of the dilute regime may have a surprisingly large effect on the overall solution of the nonlinear equations of saturation. Indeed, a fluctuation in the dilute regime may grow exponentially and thus modify its contribution to the overall amplitude. Hence, in order to enlarge our understanding of the QCD evolution with rapidity, it seems important to give a quantitative description of the fluctuation pattern generated by the BFKL evolution equations for the set of cascading dipoles (and thus gluons) near saturation, which is the goal of our paper. We shall work in the leading order in $1 / N_{c}$, where the QCD dipole framework is valid 11, 12.

The equations for the generating functionale of moments of the QCD dipole distributions have been derived already some time ago [11] and they have been used in instructive numerical simulations of onium-onium scattering [13] and also applied recently in diffractive and non-diffractive deep-inelastic scattering [14]. However, to our knowledge, a thorough investigation of their solutions for the pattern of dipoles or equivalently for the event-by-event properties of gluon transverse momenta has not yet been considered. We will provide an answer to this question in the QCD dipole approximation (and moreover in the diffusive approximation of the 1-dimensional BFKL kernel, see further on), and the results appear quite rich already within this approximation scheme. Many aspects we find show "universality" features and thus are expected to be valid also in the dipole framework beyond the approximations (going beyond the dipole approximation is still a conceptual challenge [15]).

The strategy of the present paper is to determine the structure of the event-by-event gluon transverse-momentum

† URA 2306, unité de recherche associée au CNRS. 
distributions. In the present paper, and as a first step towards a more complete solution, we will simplify the problem, focusing on modeling the QCD gluon cascading using the diffusive approximation of the BFKL kernel and aiming at a quantitative understanding of the transition-to-saturation phase within this scheme.

We show that one can build a mapping between the energy evolution of the set of gluon momenta (in the 1dimensional approximation) and the time evolution of directed polymers on a tree with disorder. This is obtained through the matching of the partition function of the polymer problem with a related event-by-event physical quantity defined from the cascading gluon process. Then we use the relation between the cascading evolution around the unitarity limit of the amplitude and the saturation mechanism to find that a spin glass structure of the gluon phase space exists at saturation, in direct analogy with the low-temperature phase of the directed polymers. We then derive the physical consequences of the spin-glass phase for the distribution of gluon transverse momenta at saturation in our model of cascading.

The plan of our study is the following. In section 2 , we shall recall the main ingredients of the rapidity evolution of the cascading dipoles and gluon-momentum distributions as driven by the BFKL evolution in the 1-dimensional and diffusive approximations. In section 3, we introduce the mapping between the cascading of gluons and the propagation of directed polymers on random trees. In section 4 we recall the nontrivial properties of the directed polymer problem, characterized by a spin-glass transition at low temperature. In section 5 , we provide a check of the consistency of the gluon/polymer mapping with the saturation mechanism in the mean field approximation. In section 6 , we derive the gluon phase space properties at large rapidity from the spin-glass features. The final section 7 is devoted to conclusion and outlook.

2. In this section, we present some known results on the equations governing the dipole distributions in the BFKL framework together with those concerning the saturation amplitude in the mean-field approximation and relevant for our study.

\section{QCD rapidity evolution}

Let us start by briefly describing the QCD evolution of the dipole distributions in the Balitsky-Fadin-Kuraev-Lipatov BFKL framework 11, 13, 14].

Formally, let us denote $\mathcal{Z}_{\mathbf{v w}}[Y, U]$ the generating functional of moments for the N-uple dipole-probability distributions after a rapidity evolution range $Y$. The dipole probabilities can be deduced from $\mathcal{Z}_{\mathbf{v w}}[Y, U]$ by functionally differentiating with respect to the source fields $U$ and then letting $U \rightarrow 0$. the evolution equation satisfied by $\mathcal{Z}_{\mathbf{v w}}[Y, U]$ reads

$$
\frac{\partial \mathcal{Z}_{\mathbf{v w}}}{\partial Y} \equiv \int_{z} d^{2} z \mathcal{K}(\mathbf{v}, \mathbf{w} ; \mathbf{z})\left\{\mathcal{Z}_{\mathbf{v z}} \mathcal{Z}_{\mathbf{z w}}-\mathcal{Z}_{\mathbf{v w}}\right\}
$$

where

$$
\mathcal{K}(\mathbf{v}, \mathbf{w} ; \mathbf{z})=\frac{\alpha_{s} N_{c}}{\pi} \frac{(\mathbf{v}-\mathbf{w})^{2}}{(\mathbf{v}-\mathbf{z})^{2}(\mathbf{z}-\mathbf{w})^{2}}
$$

is the BFKL kernel [1] describing the dissociation vertex of one dipole $(\mathbf{v}, \mathbf{w})$ into two dipoles at ( $\mathbf{v}, \mathbf{z})$ and $(\mathbf{z}, \mathbf{w})$, where $\mathbf{v}, \mathbf{w}, \mathbf{z}$ are arbitrary 2-dimensional transverse space coordinates .

From Eq.(1), we will mainly retain its physical meaning. The functionale structure of Eq.(1) describes a 2dimensional tree structure of dipoles in transverse position space evolving with rapidity. Let us, for instance, focus on the rapidity evolution starting from one massive $q \bar{q}$ pair or onium [1]], see Fig 1 At each branching vertex, the wave function of the onium-projectile is described by a collection of color dipoles. The dipoles split with a probability per unit of rapidity defined by the BFKL kernel (2). The BFKL rapidity evolution generates a cascade of dipoles. Each vertex of the cascade is governed in coordinate space by the 2-dimensional BFKL kernel (2), determining both the energy and transverse position space evolution of the cascade, as sketched in Fig 1 As we shall see later on, this cascading property can be converted in a similar cascade of gluons in momentum space.

At this stage, it is interesting to note [14] the mathematical similarity of (11) with the BK equation [5], as verified by the S-Matrix element of the dipole-target amplitude $\mathrm{S}(\mathbf{v}, \mathbf{w}, \mathrm{Y})$ in the mean-field approximation. The equation is indeed formally the same if one replaces term by term the functional $\mathcal{Z}_{\mathbf{v w}}[Y, U]$ by the function $\mathcal{S}(\mathbf{v}, \mathbf{w}, Y)$ representing the S-Matrix element. 


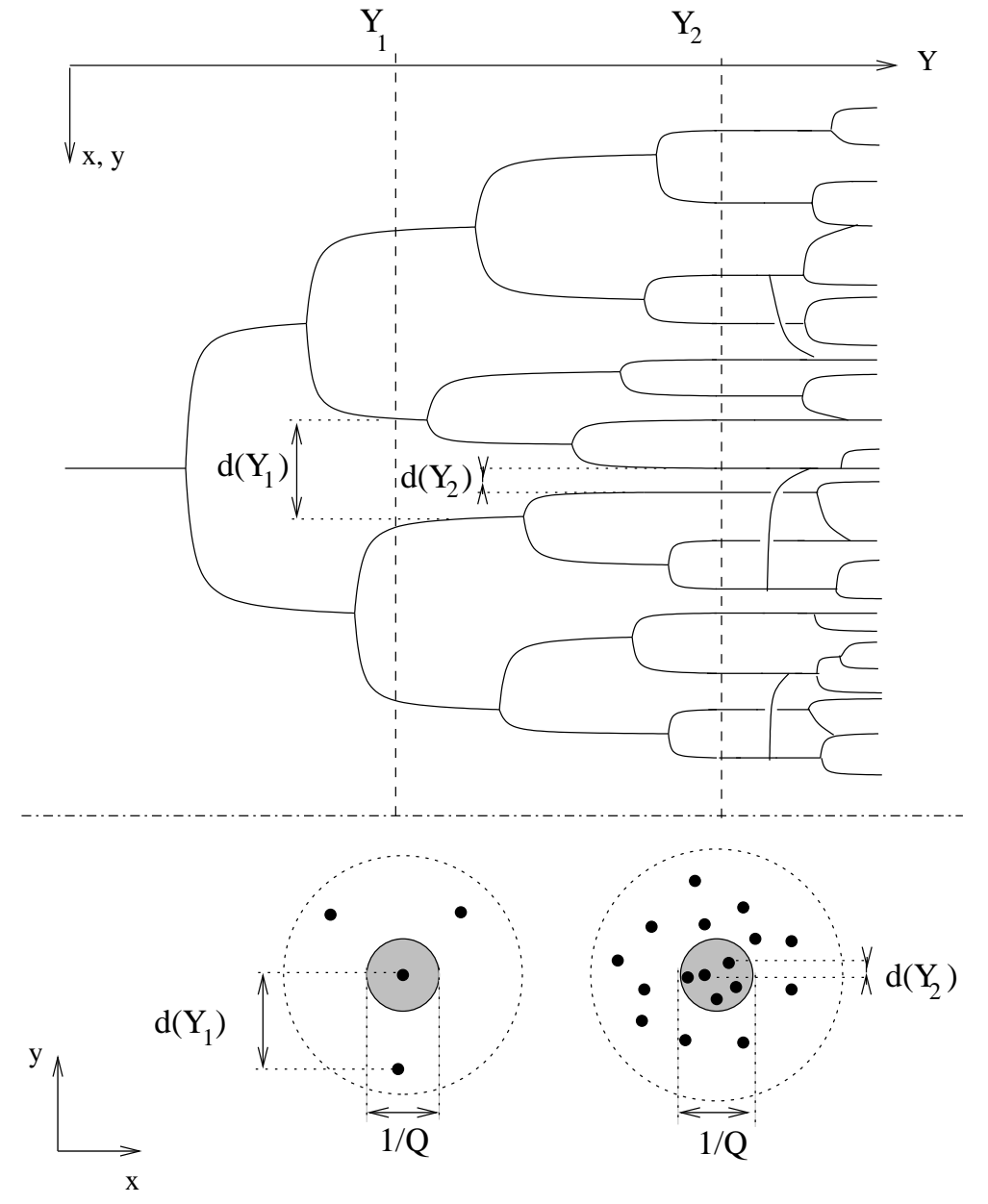

FIG. 1: BFKL cascading and saturation. The QCD branching process in the BFKL regime and beyond is represented along the rapidity axis (upper part). Its 2-dimensional counterpart in transverse position space is displayed at two different rapidities (lower part). The interaction region is represented by a shaded disk of size $1 / Q$. At rapidity $Y_{1}$, the interaction still probes individual dipoles (or gluons), which corresponds to the exponential BFKL regime. There exists a smooth transition to a regime where the interaction only probes groups of dipoles or gluons, e.g. at rapidity $Y_{2}$. This gives a description of the near-saturation region corresponding to a mean-field approximation, where correlations can be neglected. Further in rapidity, $Y>Y_{2}$, other dynamical effects, such that merging and correlations appear, leading eventually to the CGC.

\section{Gluons in the 1-dimensional and diffusive approximation}

As an approximation of the 2-dimensional formulation of the BFKL kernel (2), obtained when one neglects the impact-parameter dependence, we shall restrict our analysis in the present paper to the 1-dimensional reduction of the problem to the transverse-momenta squared $k_{i}^{2}$ of the cascading gluons. After Fourier transforming to transversemomentum space, the leading-order BFKL kernel [1] defining the rapidity evolution in the 1-dimensional approximation is known [5] to act in transverse momentum space as a differential operator of infinite order

$$
\chi\left(-\partial_{l}\right) \equiv 2 \psi(1)-\psi\left(-\partial_{l}\right)-\psi\left(1+\partial_{l}\right)
$$

where $l=\log k^{2}$ and $Y$ is the rapidity in units of the fixed coupling constant $\frac{\alpha_{s} N_{c}}{\pi}$.

In the sequel, we shall restrict further our analysis to the diffusive approximation of the BFKL kernel. We thus expand the BFKL kernel to second order around some value $\gamma_{c}$

$$
\chi(\gamma) \sim \chi_{c}+\chi_{c}^{\prime}\left(\gamma-\gamma_{c}\right)+\frac{1}{2} \chi_{c}^{\prime \prime}\left(\gamma-\gamma_{c}\right)^{2}=A_{0}-A_{1} \gamma+A_{2} \gamma^{2}
$$

where $\gamma_{c}$ will be defined in such a way to be relevant for the near-saturation region of the BFKL regime. 
Within this diffusive approximation, it is easy to realize that the first term $\left(A_{0}\right)$ is responsible for the exponential increase of the BFKL regime while the third term $\left(A_{2}\right)$ is a typical diffusion term. The second term $\left(A_{1}\right)$ is a "shift" term since it amounts to a rapidity-dependent redefinition of the kinematic variables, as we shall see.

In Eq.(44), $\gamma_{c}$ is chosen in order to ensure the validity of the kernel (4) in the transition region from the BFKL regime towards saturation. Indeed, the derivation of asymptotic solutions of the BK equation [ 6 ] leads to consider the condition

$$
\chi\left(\gamma_{c}\right)=\gamma_{c} \chi^{\prime}\left(\gamma_{c}\right)
$$

whose solution determines $\gamma_{c}$.

This condition applied to the kernel formula (3) gives $\gamma_{c}=\sqrt{A_{0} / A_{2}} \approx 0.6275 \ldots$ and $\left\{A_{0}, A_{1}, A_{2}\right\} \approx$ $\{9.55,25.56,24.26\}$. It is worth noting that a more general choice of the $A_{i}$ 's can be considered for the forthcoming discussion, leaving the possibility of considering also "effective" kernels [16], eventually taking into account next leading corrections and thus other values of $\gamma_{c}$.

\section{Saturation in the mean-field approximation}

As already noticed [14], the 2-dimensional BK equation describing saturation in the mean-field approximation is formally similar to (II) by substituting $\mathcal{Z}$ by $\mathcal{S}$, the S-matrix element of the scattering amplitude. In the 1-dimensional approximation which we will use, the BK equation reads

$$
\partial_{Y} T(l, Y)=\chi\left(-\partial_{l}\right) T(l, Y)-T^{2}(l, Y),
$$

with the same notations as in (31).

In the 1-dimensional approximation, the Fourier transform of the dipole-target scattering amplitude $T(l, Y)$ is related to the unintegrated gluon distribution in the target (by a simple convolution). Applying only the linear operator (3) to $T(l, Y)$ leads to an exponential increase of the gluon density with rapidity characteristic of the BFKL regime. The damping term in Eq.(6) is responsible for the saturation mechanism in the mean-field approximation.

There exists an already long literature on the properties of the BK equation (see, for instance, 3, 5, 6]). For further purpose, we remark that many results which can be obtained from the solutions of the BK equation can already be derived from the BFKL evolution when only the linear operator in Eq. (6) is retained. The idea [18, 19] is to consider the extension of the BFKL evolution in the rapidity region when the unitarity limit is reached and assume a smooth transition to saturation. This amounts to study the BFKL regime alongside the saturation line defined by the saturation scale $Q_{s}(Y)$ in the 2-dimensional $k^{2}, Y$ plane.

Indeed, one obtains a significant part [18, 19] of the results obtained [6] by a direct derivation of the asymptotic solutions of the BK equation. Let us list some of the most relevant results of this approach based on the unitarity bound: The gluon momentum distribution can be derived at high rapidity and large enough $k$. One finds

$$
T(l, Y) \propto \log \left[\frac{k^{2}}{Q_{s}^{2}(Y)}\right]\left(\frac{k^{2}}{Q_{s}^{2}(Y)}\right)^{-\sqrt{A_{0} / A_{2}}} \exp \left\{-\frac{1}{A_{2} Y} \log ^{2}\left[\frac{k^{2}}{Q_{s}^{2}(Y)}\right]\right\} .
$$

The saturation scale $Q_{s}^{2}(Y)$ is given by

$$
\log Q_{s}^{2}(Y) \propto\left[2 \sqrt{A_{0} A_{2}}-A_{1}\right] Y-\frac{3}{2 \sqrt{A_{0} / A_{2}}} \log Y+\mathcal{O}(1) .
$$

Furthermore, at large rapidity, the third term of (17) and the subasymptotic correction in (8) become negligible, leaving a gluon momentum distribution exhibiting geometric scaling [20]

$$
T(l, Y) \rightarrow T\left(\tau=\left[\frac{k^{2}}{Q_{s}^{2}(Y)}\right]\right) \propto \tau^{-\sqrt{A_{0} / A_{2}}} \log \tau ; \quad \log Q_{s}^{2}(Y) \propto\left[2 \sqrt{A_{0} A_{2}}-A_{1}\right] Y .
$$

The asymptotic results (9) can be directly obtained from the unitarity bound applied to the solution of BFKL equation [18], while the subasymptotic results can also be obtained from the BFKL equation, but with more care taken about the boundary conditions [19].

3. Now we introduce the mapping from the BFKL regime of QCD to the thermodynamics of directed polymers. As we have mentionned earlier, the BFKL rapidity evolution generates a cascade of gluons [1]. Each vertex of the 
cascade is governed in coordinate space by the 2-dimensional BFKL kernel, determining together the energy and the transverse space evolution of the cascade, see Fig 1 In transverse-momentum space and within the 1-dimensional diffusive approximation (4), we already noticed that the BFKL kernel models boils down to a branching, shift and diffusion operator acting in the gluon transverse-momentum-squared space. Hence the cascade of gluons can be put in correspondence with a continuous branching, velocity-shift and diffusion probabilistic process whose probability by unit of rapidity is defined by the coefficients $A_{i}$ of (4).

Let us first introduce the notion of gluon-momenta "histories" $k_{i}(y)$. They register the evolution of the gluonmomenta starting from the unique initial gluon momentum $k(0)$ and terminating with the specific $i$-th momentum $k_{i}$, after successive branchings. They define a random function of the running rapidity $y$, with $0 \leq y \leq Y$, the final rapidity range when the evolution ends up (say, for a given total energy). It is obvious that two different histories $k_{i}(y)$ and $k_{j}(y)$ are equal before the rapidity when they branch away from their common ancestor, see Fig 1

We then introduce random paths $x_{i}(t)$ using formal space and time coordinates $x, t, 0 \leq t \leq \Delta t$ which we relate to gluon-momenta "histories" as follows:

$$
y=\frac{t}{A_{0}} ; \quad \log k_{i}^{2}(y) \equiv-\beta\left(x_{i}(t)-x(0)\right)+\left(A_{0}-A_{1}\right) y
$$

where $\left(A_{0}-A_{1}\right) y$ is a conveniently chosen and deterministic "drift term", $x(0)$ is an arbitrarily fixed origin of an unique initial gluon and thus the same for all susequent random paths. The random paths $x_{i}(t)$ are generated by a continuous branching and Brownian diffusion process in space-time (cf. Fig 2).

As we shall determine later on, the important parameter $\beta$, which plays the rôle of an inverse temperature for the Brownian process, will be fixed to

$$
\beta=\sqrt{\frac{2 A_{2}}{A_{0}}}
$$

In fact, the relation (11) will be required by the condition that the stochastic process of random paths describes the BFKL regime of QCD near saturation. Another choice of $\beta$ would eventually describe the same branching process but in other conditions. Hence the condition (11) will be crucial to determine the QCD phase at saturation (within the diffusive approximation).

Let now introduce the tree-by-tree random function defined as the partition function of the random paths system

$$
Z(t) \equiv \sum_{i=1}^{n} e^{-\beta x_{i}(t)}=e^{-\beta x_{0}+A_{1} y} \times \frac{\sum_{i=1}^{n} k_{i}^{2}(y)}{e^{A_{0} y}}
$$

Using the fact that the total number of momenta in one event at rapidity $y$ is $n \approx\langle n\rangle=e^{A_{0} y}$ at large $y$, (12) takes the form

$$
Z=\lambda e^{A_{1} y} \times \bar{k}^{2}(y)
$$

where $\frac{1}{n} \sum_{i=1}^{n} k_{i}^{2}(y) \equiv \bar{k}^{2}(y)$ is the event-by-event average over gluon momenta at rapidity $y$ and $\lambda=e^{-\beta x_{0}}$ is an arbitrary constant. Note that one has to distinguish $\langle\cdots\rangle$, i.e. the sample-to-sample average, from $\bar{\cdots}$ which denotes the average made over only one event.

$Z(t)$ is an event-by-event random function. The physical properties are obtained by averaging various observables over the events. Note that the distinction between averaging over one event and the sample-to-sample averaging appears naturally in the statistical physics problem in terms of "quenched" disorder: the time scale associated with the averaging over one random tree structure is different (much shorter) than the one corresponding to the averaging over random trees.

In parallel with the statistical physics problem [21], it is convenient to introduce the generating functional $G(t, x)$ of its event-averaged moments $\left\langle Z^{p}\right\rangle$, namely

$$
G(t, x) \equiv \sum_{p} \frac{1}{p !}\left\langle\left[-e^{\beta x} Z(t)\right]^{p}\right\rangle=\left\langle\exp \left[-e^{-\beta x} Z(t)\right]\right\rangle=\left\langle\exp \left[-\lambda \bar{k}^{2}(y) / \kappa^{2}\right]\right\rangle
$$

where

$$
y=\frac{t}{A_{0}}, \quad \log \kappa^{2}=\beta x+\frac{A_{1}}{A_{0}} t
$$


The moments $\left\langle Z^{p}(t)\right\rangle$ are easily recovered by differentiation over $x$ or $\log \kappa^{2}$ at fixed $t$.

It is interesting to note the final form of (14) showing a similarity [14] with a unitary S-Matrix amplitude if $\kappa$ is interpreted as the scale of a transverse-momentum probe, such as depicted in Fig 11 This similarity will be useful for the derivation of saturation properties from the BFKL regime in the unitarity limit.

4. Now that we have established the connection (10) between the model of gluon-momenta submitted to branching, shift and diffusion, and the random paths defined by $x_{i}(t)$, let us rederive the properties of QCD gluon cascading near saturation from the point-of-view of the statistical random-path system. For this sake, we recall the corresponding model for directed polymers on a tree, as defined in Ref. 21].

Directed polymers on a random tree are modeled, see Fig 2 by the time evolution of random paths in 1-dimensional space with continuous diffusion and branching (with probability per unit of time fixed respectively to $\frac{1}{2}$ and 1 , by convention).

Indeed, contemplating the definitions (12) and (14) it is straightforward to identify $Z$ as the sample-to-sample partition function and $G(t, x)$ as the generating fonctional of its sample-averaged moments for the model [21] of directed polymers on a random tree.

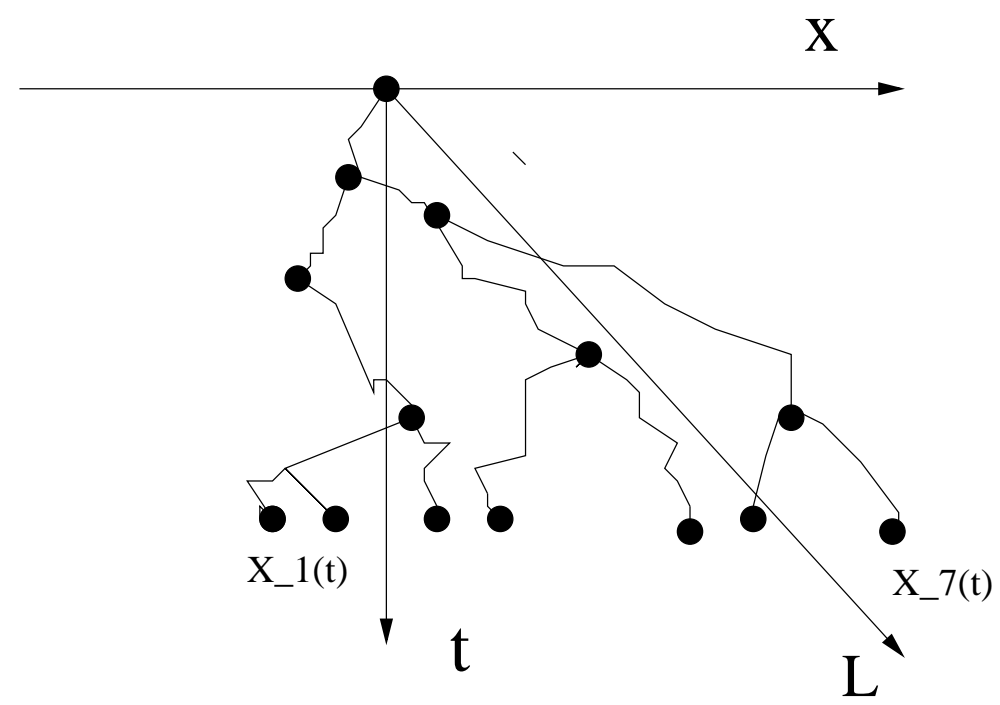

FIG. 2: Branching diffusion model for polymers. The coordinates $x_{1}(t) \cdots x_{7}(t)$ correspond to the random paths along the tree in the $x, t$ phase-space. The oblique axis is for $L=\beta x+A_{1} / A_{0} t$, which takes into account the "time-drift" in the mapping to the QCD problem.

Let us list some relevant properties of the polymer problem [21].

- $u(t, x) \equiv 1-G(t, x)$ verifies the Fisher and Kolmogorov Petrovsky Piscounov (F-KPP) equation [7]

$$
\frac{\partial}{\partial t} u(t, x)=\frac{1}{2} \frac{\partial^{2} u(t, x)}{\partial x^{2}}+u(t, x)-u^{2}(t, x) \text {. }
$$

$u(t, x)$ admits traveling-wave solutions at large time $t[8]$

$$
u(t, x) \sim u\left(x-m_{\beta}(t)\right) \approx u(x-c(\beta) t),
$$

where the wave speed $c(\beta)$ is governed by the initial condition $u\left(t_{0}, x\right) \sim e^{-\beta x}$ at large $x$. The range of values of $c(\beta)=1 / \beta+\beta / 2$ is bounded from below by $c\left(\beta_{c}\right) \equiv c=\sqrt{2}$, where the wave velocity remains fixed whenever $\beta \geq \beta_{c}=\sqrt{2}$. $c$ and $\beta_{c}$ are usually called, respectively, the critical velocity and the critical slope of the waves. In the "supercritical" regime $\beta \geq \beta_{c}$, the wave front and its speed remain "frozen" and

$$
m_{\beta}(t)=c\left(t-\frac{3}{4} \log t\right)+\mathcal{O}(1) .
$$

- The free energy of the polymer system at equilibrium, defined by

$$
F=-\frac{1}{\beta}\langle\log Z(t)\rangle \equiv-m_{\beta}(t),
$$


has a "frozen" density $F / t \rightarrow-c$ for $\beta \geq \beta_{c}=\sqrt{2}$ indicating the existence of a phase transition at $\beta_{c}$. This is related to the dynamical transition $c(\beta) \rightarrow c$ in the traveling-wave properties [8].

- Introducing the tree-by-tree free energy spectrum

$$
f(t)=-\frac{1}{\beta} \log Z(t)+\frac{1}{\beta}\langle\log Z(t)\rangle,
$$

its probability distribution $\mathcal{P}_{\beta}(f)$ around the average free energy $F$ is found by deriving asymptotic expressions for the moments [21] to satisfy

$$
\begin{aligned}
& \mathcal{P}_{\beta}(f) \sim-f e^{\sqrt{2} f} \quad \text { for } \quad f \rightarrow-\infty \\
& \mathcal{P}_{\beta}(f) \sim e^{-[(2-\sqrt{2}) f]} \quad \text { for } \quad f \rightarrow \infty,
\end{aligned}
$$

where we quoted only the distribution obtained for the supercritical regime $\beta \geq \beta_{c}$. This means that the fluctuations of the free energy around its event average remain finite even at asymptotic time, i.e. there is no thermodynamical self-averaging, demonstrating that the phase transition is not similar to an ordinary liquid-gas phase transition.

- The corresponding phase transition is towards a spin-glass phase characterized by a non-trivial structure in "valleys" or ground state minima, which can be explored quite in detail. We shall describe this structure in the next section, since it will give, through matching with the gluon cascading problem, a description of the gluon-momentum phase at saturation within the diffusive mean-field scheme.

5. Let us make now the connection of the directed-polymer properties with the description of the gluon-momentum phase near saturation.

Our aim is to study the structure of the gluon-momentum spectrum when the system reaches saturation. We thus start with a simple initial state (e.g. an onium or one gluon) and, the rapidity increasing, the state develops into a cascading structure of gluons governed by the model that we assume. It consists in random tree structures generated by branching, shifting and diffusing, as previously described. As we have discussed in introduction, the rapid exponential increase of the number of gluons generates reinteractions which damp the exponential increase and cause the saturation phenomenon [3].

In the simple mean-field approximation which neglects the dynamical correlations [5], the saturation mechanism is known to be studied from the BFKL properties in the following approximation 18. When the exponential increase of the gluon density reaches the unitarity limit of the amplitude, the saturation effects set in. This defines a kinematical region in the $k, Y$ plane around the saturation scale $Q_{s}(Y)$ where the BFKL regime is expected to smoothly connect the saturation region. Indeed, this expectation has been verified for the amplitude, where many aspects of the saturation features rigorously found $[\underline{6}]$ for the solutions of the mean field BK saturation equation have been already derived from the BFKL regime considered near the unitarity limit [18].

We shall now try and extend this approach of saturation, which provided already valuable results on averaged observables, to the event-by-event spectrum of gluon transverse momenta. In other words, we study momenta of the order of the saturation scale, i.e. $k_{i}^{2}=\mathcal{O}\left(Q_{s}^{2}\right)$. This is nothing else than the neighbourhood of the kinematic region where "universal" traveling-wave symptotic solutions of the BK equation exist [6]. We thus expect from the studies of Ref. [6] that the properties we shall derive will be independent of initial conditions (provided they are leading to a "supercritical" regime), of the precise form of the kernel (beyond the diffusive approximation) and even of the precise form of the non-linearities [8].

A comment of caution is nevertheless in order here, concerning the domain of validity of our appoach. The properties in the region of smaller momenta $k_{i} / Q_{s} \ll 1$ will probably depend more on the specific structure of the BFKL kernel beyond the diffusive approximation while the region with $k_{i} / Q_{s} \gg 1$ will keep the memory of the initial conditions. They should require a different treatment, which is not included in our present work and deserves later study.

Inserting the QCD definitions (13) in (19) for observables after a final rapidity evolution-range $Y$ (or $\Delta t$ in time) gives

$$
\begin{aligned}
\left\langle\log \bar{k}^{2}\right\rangle & \equiv\langle\log Z\rangle-\log \lambda-A_{1} Y=\beta m_{\beta}(\Delta t)-A_{1} Y-\log \lambda \\
& =2 \sqrt{\frac{A_{2}}{A_{0}}}\left(A_{0} Y-\frac{3}{4} \log Y\right)-A_{1} Y+\mathcal{O}(1) \simeq \log Q_{s}^{2}
\end{aligned}
$$


The second line of Eqs.(22) is obtained by identifying the inverse temperature $\beta$ with its value defined by the relation (11). This the condition to verify $\bar{k}^{2} \approx Q_{s}^{2}$, the identification coming from formula (8). This condition is crucial to check the main saturation property that the sample averaging gluon momentum stays at the value of the saturation scale. Hence, the relations (22), important for the QCD saturation problem, determines the equivalent temperature of the polymer system. Starting from (4) and (11), the inverse temperature obtains $\beta=1 / \mu \approx 2.25>\beta_{c}=\sqrt{2}$ which is larger than the critical value. As a consequence, we are led to consider and study the low-temperature phase of the equivalent random-path system in order to get information on the QCD phase of gluons near saturation.

As a further check of the validity of the random cascading model in the "unitarity limit" region, where the average momentum is consistently found to be determined by the saturation scale, let us discuss the distribution around the average.

The event-by-event mean momentum $\bar{k}^{2}$ at rapidity $Y$ can be read off from Eq.(20) as being

$$
\log \left[\frac{\bar{k}^{2}}{Q_{s}(Y)^{2}}\right] \equiv-\beta f(\Delta T)=-\sqrt{\frac{2 A_{2}}{A_{0}}} f\left(A_{0} Y\right)
$$

and, from the first line of (21), when $e^{-\beta f} \sim \bar{k}^{2} / Q_{s}^{2}(Y)$ is large, its probability distribution is given by

$$
\mathcal{P}\left(\log \left[\frac{\bar{k}^{2}}{Q_{s}^{2}}\right]\right) \propto \log \left[\frac{\bar{k}^{2}}{Q_{s}^{2}}\right] \exp \left\{-\sqrt{\frac{A_{0}}{A_{2}}} \log \left[\frac{\bar{k}^{2}}{Q_{s}^{2}}\right]\right\} .
$$

By comparison with formula (9), the interpretation of Eq. (24) is clear. The probability law (24) for the event-byevent partition function coincides with the gluon distribution in the saturation region and follows geometric scaling. This gives the confirmation, through the properties of the directed polymers, that the gluon-cascading model near the unitarity limit gives the correct saturation scale and gluon distribution. In mathematical terms, this comes from the fact that the equation for the generating function of moments for the model (16) is in the same (FKPP) universality class than the BK equation [6].

Both properties (22), (24) prove the consistency of the model with the properties expected from saturation. We shall then look for other properties of the cascading gluon model. It is important to realize that this consistency fails for any other choice of the parameter $\beta$ different from (11). This justifies a-posteriori the identification of the equivalent temperature of the system in the gluon/polymer mapping framework.

6. Let us now come to the main topics concerning the determination of structure of the gluon-momentum phase at saturation in the diffusive model.

The striking property of the directed polymer problem on a random tree is the spin-glass structure of the low temperature phase. As we shall see this will translate directly into a specific clustering structure of gluon transverse momenta in their phase near the "unitarity limit". Let us divide the discussion in a few subsections.

i) The equivalent temperature. Following the relation (11), one is led to consider the polymer system at temperature $T$ with

$$
\frac{T_{c}-T}{T_{c}} \equiv 1-\frac{\beta_{c}}{\beta}=1-\gamma_{c},
$$

where $\gamma_{c}$ is the critical exponent. We are thus naturally led to consider the low-temperature phase $\left(T<T_{c}\right)$, at some distance $T_{c}-T$ from the critical temperature $T_{c}=1 / \sqrt{2}$. In the language of traveling waves $[\underline{8}$, this corresponds to a "pulled-front" condition with "frozen" and "universal" velocity and front profile.

ii) The asymptotic phase landscape. As derived in [21], the phase space of the polymer problem is structured in "valleys" which are in the same universality class as those of the Random Energy Model (REM) [22] and of the infinite range Sherrington-Kirkpatrick (SK) model [23].

Translating these results in terms of gluon momenta (in modulus), the phase space landscape consists in eventby-event distribution of clusters of momenta around some values $k_{s i}^{2} \equiv 1 /\left(n_{i}\right) \quad \sum_{i \in s i} k_{i}^{2}$, where $n_{i}$ is the cluster multiplicity. The probability weights to find a cluster $s_{i}$ after the whole evolution range $Y$ is defined by

$$
W_{s i}=\frac{\sum_{i \in s i} k_{i}^{2}}{\sum_{i} k_{i}^{2}}
$$

where the summation in the numerator is over the momenta of gluons within the $s_{i}^{\text {th }}$ cluster (see Fig 3 ). The normalized distribution of weights $W_{s i}$ thus allows one to study the probability distribution of clusters. The clustering tree structure, (called "ultrametric" in statistical mechanics) is the most prominent feature of spin-glass systems [24]. 
Note again that, for the QCD problem, this property is proved for momenta in the region of the "unitarity limit", or more concretely in the momentum region around the saturation scale. This means that the cluster average-momentum is also such that $k_{s i}^{2}=\mathcal{O}\left(Q_{s}^{2}\right)$. Hence the clustering structure is expected to appear in the range which belongs to the traveling-wave front $[\underline{6}]$ or, equivalently, of clustering with finite fluctuations around the saturation scale.

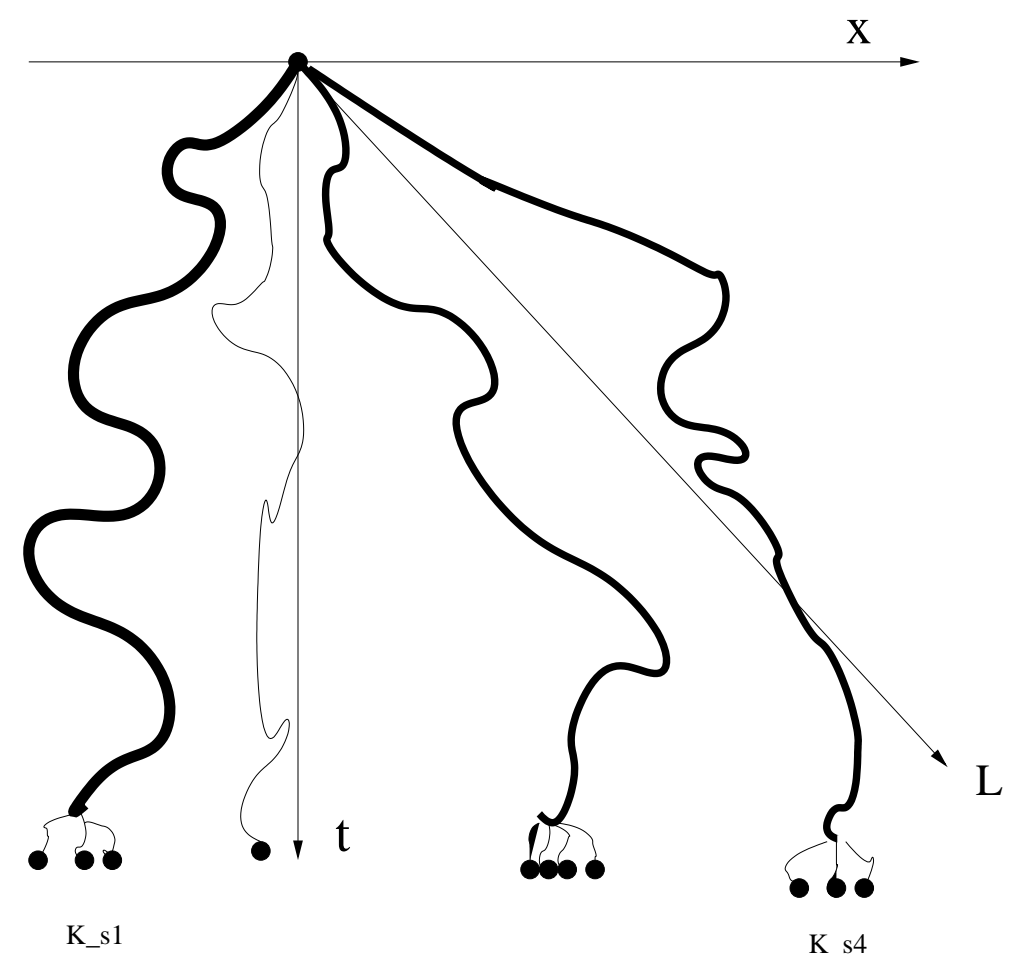

FIG. 3: The clustering structure. The drawing represents the $s_{1} \cdots s_{4}$ clusters near momenta $k_{s 1} \cdots k_{s 4}$. They branch either near $t \ll 1$ or $(\Delta t-t) \ll 1$, where $\Delta t$ is the total amount of time evolution.

iii) The "overlap" function. There exists in statistical physics of spin-glasses a well-known function which reveals the space-time structure of a spin-glass state in a quantitative way: the "overlap" function 24]. Translating the definitions (cf. 21] ) in terms of the QCD problem, one introduces the overlap between two final gluon momenta $k_{i}$ and $k_{j}$, using their "histories" $k_{i}(y)$ and $k_{j}(y)$. We recall that $k_{i}(y)$ and $k_{j}(y)$ defined as function of the running rapidity $y$ correspond to the two (not always disjoint) paths histories which finally lead to the momenta $k_{i}$ and $k_{j}$ observed at final (and large) rapidity $Y$.

The overlap is defined by the quantity $Q_{i j}$, the fraction of the total rapidity $Y$ for which $k_{i}(y)=k_{j}(y), \quad 0 \leq Q_{i j} \leq 1$. It is a measure of the rapidity interval ("time") during which they belong to the same branch before splitting. For a given event, the probability $\tilde{\mathcal{Y}}(q)$ of finding an overlap $q$ can be defined by

$$
\tilde{\mathcal{Y}}(q) d q=\frac{1}{\left\{\sum\left(k_{i}^{2}\right)\right\}^{2}} \sum_{i, j=1}^{n} k_{i}^{2} k_{j}^{2} \Theta\left(q<Q_{i j}<q+d q \Longleftrightarrow k_{i}(q Y)=k_{j}(q Y) \text { in }[q, q+d q]\right),
$$

where the characteristic function $\Theta$ restricts the sum to the pairs of momenta which where on the same branch in the interval of rapidity $[q, q+d q]$. The result reads (from Ref.[21])

$$
\tilde{\mathcal{Y}}(q) d q=\delta(q-1) \mathcal{Y}+\delta(q)(1-\mathcal{Y}) \equiv \delta(q-1) \sum_{s i} W_{s i}^{2}+\delta(q)\left(1-\sum_{s i} W_{s i}^{2}\right),
$$

where $\mathcal{Y}=\sum_{s i} W_{s i}^{2}$ is an event-by-event indicator of the strength of clustering.

The result (27) means that, in the thermodynamical limit, the gluon momenta branch effectively either near $y=0$ or near $y=Y$ for asymptotic $Y$, see Fig 3 Hence the "microscopic" branching structure of the model of Fig 2 at finite $y$, occupies only a vanishing (when $Y \rightarrow \infty$, i.e. in the thermodynamical limit) fraction of the rapidity evolution, either at the beginning or at the end but not in the middle nor for a finite fraction of the asymptotic rapidity. 


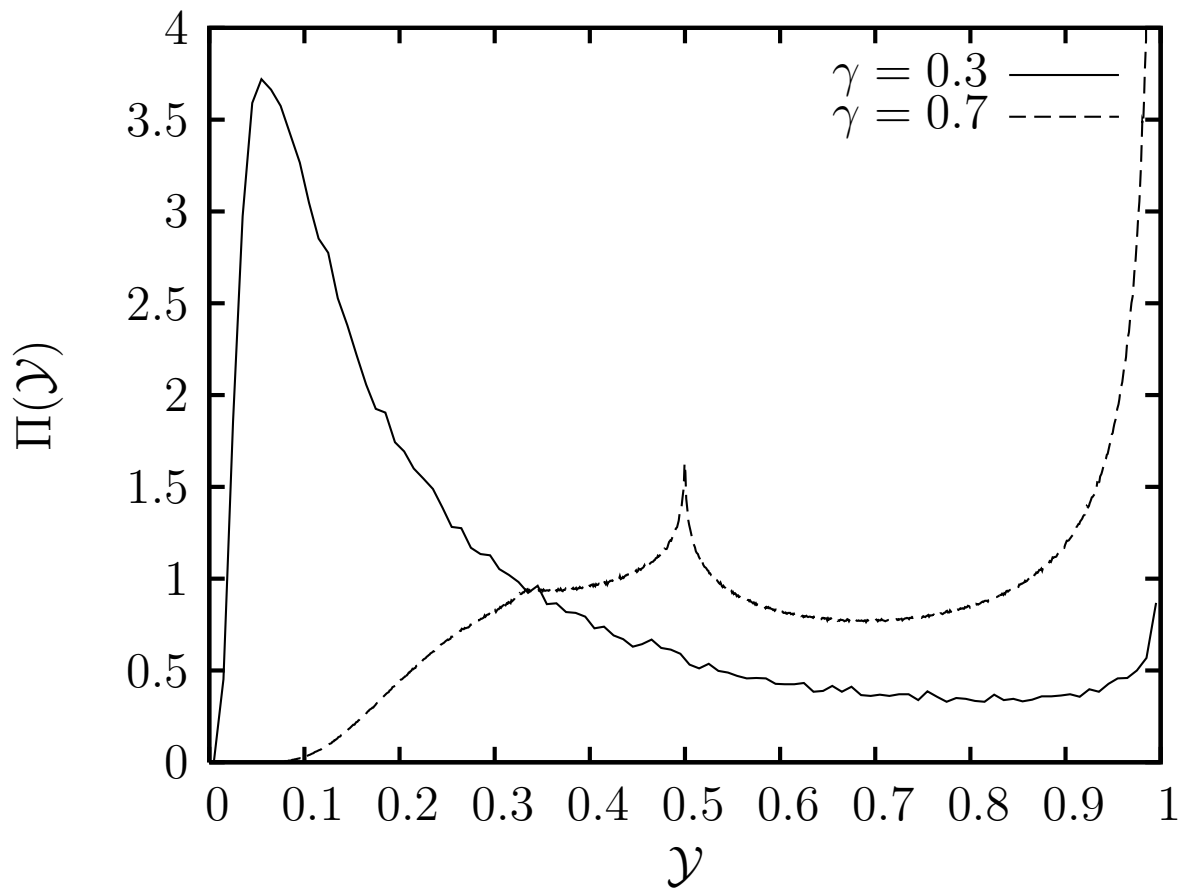

FIG. 4: The probability distribution of overlaps $\Pi(\mathcal{Y})$. The figure (the simulation is by courtesy from [29], using the method of Ref.[26]) is drawn both for the theoretical QCD value $\gamma_{c}=0.7$, and for $\gamma_{c}=0.3$ for comparison. The statistics used for the simulation is $10^{8}$ events in $10^{3}$ bins for $\gamma_{c}=0.3$ and $3.2510^{6}$ events in $10^{2}$ bins for $\gamma_{c}=0.7$.

The probability distribution of overlaps $\Pi(\mathcal{Y})$ is identical to the one of the REM and SK models and shares many qualitative similarities with other systems possessing a spin-glass phase [25, 26]. Being quite non-trivial analytically, it is more easily defined by its moments

$$
\left\langle\mathcal{Y}^{\nu}\right\rangle=\frac{(-1)^{n+1} T_{c} / T}{\Gamma(2 \nu) \Gamma(n-\nu)} \int_{0}^{\infty} d \rho \rho^{n-1-\nu} \frac{d^{n} \log g(\rho)}{d \rho^{n}} \quad \text { for } \quad n-1<\rho<n,
$$

with (see [25, 26] )

$$
g(\rho)=\int_{0}^{\infty} d v v^{-T / T_{c}-1}\left(1-e^{-v-\rho v^{2}}\right)
$$

The overlap distribution depends only on $T / T_{c}$, which confirms its high degree of universality . One finds, in particular, $\langle\mathcal{Y}\rangle=1-T / T_{c}$, which is the average probability of having overlap 1 , while $T / T_{c}$ is the probability of overlap 0.

The rather involved probability distribution $\Pi(\mathcal{Y})$ is quite intringuing, as obtained by inversion of moments $\left\langle\mathcal{Y}^{\nu}\right\rangle$ 25, 26]. It possesses apriori an infinite number of singularities at $\mathcal{Y}=1 / n, n$ integer. It can be seen when the temperature is significantly lower from the critical value, see for instance the curve for $1-T / T_{c}=.7$ in Fig 4 However, the predicted curve for the QCD value $1-T / T_{c}=.3$ is smoother and shows only a final cusp at $W_{s}=1$ within the considered statistics. It thus seems that configurations with only one cluster can be more prominent than the otherwise smooth generic landscape. However, it is also a "fuzzy" landscape since many clusters of various sizes seem to coexist in general.

iv) The cluster distribution. The sample-to-sample distribution of "valleys" can be explicitely computed by a generalization of the overlap function [25, 26] to any power $\epsilon, \sum W_{s i}^{\epsilon}$, which translates into a well-defined distribution of cluster-weight probability. For instance one determines:

$$
\begin{aligned}
\Phi(W) & =\frac{W^{-\gamma_{c}-1}(1-W)^{\gamma_{c}-1}}{\Gamma\left(\gamma_{c}\right) \Gamma\left(1-\gamma_{c}\right)} \\
\Phi\left(W_{1}, W_{2}\right) & =\gamma_{c} \frac{\left(W_{1} W_{2}\right)^{-\gamma_{c}-1}\left(1-W_{1}-W_{2}\right)^{2 \gamma_{c}-1}}{\Gamma\left(2 \gamma_{c}\right) \Gamma^{2}\left(1-\gamma_{c}\right)} \times \theta\left(1-W_{1}-W_{2}\right) \\
\Phi\left(W_{1}, W_{2}, \cdots, W_{p}\right) & =\gamma_{c}^{p} \Gamma(p) \frac{\left(W_{1} W_{2} \cdots W_{p}\right)^{-\gamma_{c}-1}\left(1-\sum_{1}^{p} W_{i}\right)^{p \gamma_{c}-1}}{\Gamma\left(p \gamma_{c}\right) \Gamma^{p}\left(1-\gamma_{c}\right)} \times \theta\left(1-\sum_{1}^{p} W_{i}\right),
\end{aligned}
$$


for, respectively, the average number $\Phi(W)$ of clusters of weight $W_{s i} \equiv W$, the average number of pairs $\Phi\left(W_{1}, W_{2}\right)$ of clusters in the same event with weights $W_{s i} \equiv W_{1}, W_{2}$, and more generally $\Phi\left(W_{1}, W_{2}, \cdots, W_{p}\right)$ the average number of p-uples of clusters with weights $W_{s i} \equiv W_{2}, \cdots W_{p}$.

It is interesting to note that smaller is $\gamma_{c}=T / T_{c}$, smaller is the probability to find $W_{s} \sim 0$, i.e. no clustering, and higher is the probability for having one big cluster $W_{s} \sim 1$. As an illustration, the phenomenological value $\gamma_{c} \approx 0.3$ leads to a more pronounced clustering structure than the BFKL value $\gamma_{c} \approx 0.6$. The sharp structuration in clusters expected for small $\gamma_{c}$ can already be seen in the overlap distribution of Fig 4

7. Let us summarize the main points of our approach. We investigated the landscape of transverse momenta in gluon cascading around the saturation scale at asymptotic rapidity. Limiting our study to a diffusive 1-dimensional modelization of the BFKL regime of gluon cascading, we make use of a mapping on a statistical physics model for directed polymers propagating along random tree structures at fixed temperature. We then focus our study on the region near the unitarity limit where information can be obtained on saturation, at least in the mean-field approximation. Our main result is to find a low-temperature spin-glass structure of phase space, characterized by event-by-event clustering of gluon transverse momenta (in modulus) in the vicinity of the rapidity-dependent saturation scale. The weight distribution of clusters and the probability of momenta overlap during the rapidity evolution are derived.

Interestingly enough the clusters at asymptotic rapidity are branching either near the beginning ("overlap 0" or $y / Y \ll 1$ ) or near the end ("overlap 1 " or $1-y / Y \ll 1$ ) of the cascading event. The probability distribution of overlaps is derived and shows a rich singularity structure.

Phenomenological and theoretical questions naturally emerge from this study.

On a phenomenological ground, it is remarkable that saturation density effects are not equally spread out on the event-by-event set of gluons; our study suggests that there exists random spots of higher density whose distribution may possess some universality properties. In fact it is natural to expect this clustering property to be present not only in momentum modulus (as we could demonstrate) but also in momentum azimuth-angle. This is reminiscent of the "hot spots" which were some time ago [27] advocated from the production of forward jets in deep-inelastic scattering at high energy (small Bjorken variable). The observability of the cluster distribution through the properties of "hot spots" is an interesting possibility.

On the theoretical ground, the questions arise how the pattern of fluctuations we found influence the onset of saturation and its properties, given the fact that fluctuations are of primordial importance in the development of the whole QCD process, as we have stressed in the introduction.

First, the question arises to extend the study to the full BFKL kernel (3), and in two dimensions, as given initially by Eq. (11). Universality properties of the solutions of the BK equations in the traveling-wave formalism [6] and in the 2-dimensional case 28] would encourage such a generalization. However, some care must be taken of the potential problems which can arise from an event-by-event description of the cascading regime. The suggestion is 29] to formulate the problem directly in the full 2-dimensional position space, for which the gluon cascading and the unitarity constraints are well defined. Some preliminary numerical results are encouraging.

Second, it would be instructive to incorporate the pattern of fluctuations we found in the calculation of the contribution of Pomeron loops to the scattering amplitude. The Pomeron loops are found to be important to consider and are the subject of many studies (see, e.g. 10]). The Pomeron loops are obtained by merging the splitting of cascading gluons of the projectile with the merging in the target. We think that the spin-glass structure of the cascading gluons could lead to useful hints for dealing with the Pomeron loops.

In conclusion, we think that the already rich structure of the phase space landscape of gluon clusters found with the diffusive model of gluon cascading opens an interesting way towards a deeper understanding of the QCD phase in the saturation regime and could lead to a deeper description the Color Glass Condensate.

I would like to thank Edmond Iancu for inspiring and stimulating remarks and Cyrille Marquet and Gregory Soyez for many fruitful suggestions and a careful reading of the manuscript. Special thanks are due to Gregory Soyez for

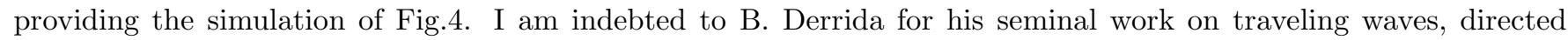
polymers and spin glasses.

* Electronic address: pesch@spht.saclay.cea.fr 
[1] L. N. Lipatov, Sov. J. Nucl. Phys. 23, 338 (1976); E. A. Kuraev, L. N. Lipatov, and V. S. Fadin, Sov. Phys. JETP 45, 199 (1977); I. I. Balitsky and L. N. Lipatov, Sov. J. Nucl. Phys. 28, 822 (1978).

[2] L.V. Gribov, E.M. Levin and M.G. Ryskin, Phys. Rep. 100, 1 (1983).

[3] L. McLerran and R. Venugopalan, Phys. Rev. D 49, 2233 (1994); 49, 3352 (1994); 50, 2225 (1994); A. Kovner, L. McLerran and H. Weigert, ibid. 52, 6231 (1995); 52, 3809 (1995); R. Venugopalan, Acta Phys.Pol. B 30, 3731 (1999); E. Iancu, A. Leonidov, and L. McLerran, Nucl. Phys. A692, 583 (2001); idem, Phys. Lett. B 510, 133 (2001); E. Iancu and L. McLerran, ibid. 510, 145 (2001); E. Ferreiro, E. Iancu, A. Leonidov and L. McLerran, Nucl. Phys. A703, 489 (2002); H. Weigert, ibid. A703, 823 (2002). For comprehensive reviews on saturation and other references, see e.g. A.H. Mueller, "Parton saturation: An overview", hep-ph/0111244 E. Iancu and R. Venugopalan, "The color glass condensate and high energy scattering in QCD," arXiv:hep-ph/0303204

[4] L. McLerran, "What is the evidence for the color glass condensate?," arXiv:hep-ph/0402137

[5] I. I. Balitsky, Nucl. Phys. B 463, 99 (1996) arXiv:hep-ph/9509348; Y. V. Kovchegov, Phys. Rev. D60, 034008 (1999), arXiv:hep-ph/9901281.

[6] S. Munier and R. Peschanski, Phys. Rev. Lett. 91, 232001 (2003) arXiv:hep-ph/0309177; Phys. Rev. D69, 034008 (2004) arXiv:hep-ph/0310357; Phys. Rev. D70, 077503 (2004) arXiv:hep-ph/0310357.

[7] R. A. Fisher, Ann. Eugenics 7, 355 (1937); A. Kolmogorov, I. Petrovsky, and N. Piscounov, Moscou Univ. Bull. Math. A1, 1 (1937).

[8] M. Bramson, Mem. Am. Math. Soc. 44, 285 (1983); E. Brunet and B. Derrida, Phys. Rev. E56, 2597 (1997); U. Ebert, W. van Saarloos, Physica D 146, 1 (2000).

[9] E. Brunet and B. Derrida, Phys. Rev. E57 (1997) 2597 arXiv:cond-mat/0005362; Comp. Phys. Comm. 121 (1999) 376 [arXix cond-mat/0005364].

[10] A. H. Mueller and A. I. Shoshi, Nucl. Phys. B 692 (2004) 175 arXiv:hep-ph/0402193; E. Iancu, A. H. Mueller and S. Munier, Phys. Lett. B 606, 342 (2005) arXiv:hep-ph/0410018. S. Munier, Nucl. Phys. A 755, 622 (2005) arXiv:hep-ph/0501149; E. Iancu and D. N. Triantafyllopoulos, Nucl. Phys. A 756 (2005) 419 arXiv:hep-ph/0411405; Phys. Lett. B 610 (2005) 253 arXiv:hep-ph/0501193. C. Marquet, R. Peschanski and G. Soyez, "Consequences of strong fluctuations on high-energy QCD evolution," arXiv:hep-ph/0512186

[11] A. H. Mueller, Nucl. Phys. B 415, 373 (1994); A. H. Mueller and B. Patel, Nucl. Phys. B 425, 471 (1994) arXiv:hep-ph/9403256; A. H. Mueller, Nucl. Phys. B 437, 107 (1995) arXiv:hep-ph/9408245.

[12] N. N. Nikolaev and B. G. Zakharov, Z. Phys. C49 (1991) 607.

[13] G. P. Salam, Nucl. Phys. B 449, 589 (1995) arXiv:hep-ph/9504284, ibid. 461, 512 (1996) arXiv:hep-ph/9509353; Comput. Phys. Commun. 105, 62 (1997) arXiv:hep-ph/9601220; A. H. Mueller and G. P. Salam, Nucl. Phys. B 475, 293 (1996) arXiv:hep-ph/9605302.

[14] For recent discussion and applications of the dipole generating functionale, see Y. Hatta, E. Iancu, C. Marquet, G. Soyez and D. N. Triantafyllopoulos, "Diffusive scaling and the high-energy limit of deep inelastic scattering in QCD at large $\mathrm{N}(\mathrm{c})$, , arXiv:hep-ph/0601150

[15] A. Kovner and M. Lublinsky, Nucl. Phys. A 767, 171 (2006) arXiv:hep-ph/0510047. E. Iancu, G. Soyez and D. N. Triantafyllopoulos, "On the probabilistic interpretation of the evolution equations with Pomeron loops in QCD," arXiv:hep-ph/0510094

[16] R. Peschanski, Phys. Lett. B 622, 178 (2005) arXiv:hep-ph/0505237; C. Marquet, R. Peschanski and G. Soyez, Phys. Lett. B 628, 239 (2005) arXiv:hep-ph/0509074.

[17] K. Golec-Biernat and M. Wusthoff, Phys. Rev. D 59, 014017 (1999) arXiv:hep-ph/9807513.

[18] E. Iancu, K. Itakura and L. McLerran, Nucl. Phys. A708, 327 (2002), arXiv:hep-ph/0203137;

[19] A. H. Mueller and D. N. Triantafyllopoulos, Nucl. Phys. B640, 331 (2002), arXiv:hep-ph/0205167.

[20] A. M. Staśto, K. Golec-Biernat, and J. Kwiecinski, Phys. Rev. Lett. 86, 596 (2001), arXiv:hep-ph/0007192.

[21] B. Derrida and H. Spohn, J. Stat. Phys. 51, 817 (1988).

[22] B. Derrida, Phys. Rev. Lett. 45, 79 (1980) Phys. Rev. B 24,2613 (1980)

[23] D. Sherrington and S. Kirkpatrick Phys. Rev. Lett. 35, 1972 (1975) Phys. Rev. B 17, 4384 (1978)

[24] The classical book on spin-glass theory and the overlap function is: M. Mézard, G. Parisi and M. A. Virasoro "Spin glass theory and beyond" World Scientific Lecture Notes in Physics, 91987.

[25] B. Derrida and G. Toulouse J. Physique Lett. 46, L223 (1985);

[26] B. Derrida and H. Flyvbjerg J. Phys. A: Math. Gen. 20, 5273 (1987).

[27] A. H. Mueller, Nucl. Phys. Proc. Suppl. B18C (1990) 125; J. Phys. G17 (1991) 1443. Jochen Bartels (Hamburg U.), M. Besancon (Saclay), A. De Roeck (Munich, Max Planck Inst.), J. Kurzhofer (Dortmund U.),. 1991, "Measurement of hot spots at HERA, In *Hamburg 1991, Proceedings, Physics at HERA, vol. 1* 203-213. (see High energy physics index 30 (1992) No. 12988). J. Bartels, "Measurement of hot spots in the proton at HERA," P.epared for International Workshop on Quark Cluster Dynamics, Bad Honnef, Germany, 29 Jun - 1 Jul 1992 J. Bartels and H. Lotter, Phys. Lett. B 309, 400 (1993).

[28] C. Marquet, R. Peschanski and G. Soyez, Nucl. Phys. A 756, 399 (2005) arXiv:hep-ph/0502020; C. Marquet and G. Soyez, Nucl. Phys. A 760, 208 (2005) arXiv:hep-ph/0504080.

[29] G.Soyez, private communication. 